\title{
Fluorofoldamer-Based Salt- and Proton-Rejecting Artificial Water Channels for Ultrafast Water Transport
}

\author{
Jie Shen, ${ }^{1}$ Arundhati Roy, ${ }^{2}$ Himanshu Joshi, ${ }^{3}$ Laxmicharan Samineni, ${ }^{4}$ Ruijuan Ye, ${ }^{1}$ Yu-Ming Tu, Woochul \\ Song, ${ }^{4}$ Matthew Skiles, ${ }^{5}$ Manish Kumar, ${ }^{4,5 *}$ Aleksei Aksimentiev, ${ }^{3 *}$ and Huaqiang Zeng ${ }^{1 *}$
}

One Sentence summary: We report here the best artificial water channel ever reported in terms of structural robustness, facile synthesis and water transport property.

Here, we report on a novel class of fluorofoldamer-based artificial water channels (AWCs) that combines excellent water over ion selectivity with extraordinarily high water transport efficiency and structural simplicity and robustness. These AWCs were produced by a facile one-pot copolymerization reaction under mild conditions. Among these channels, the best-performing channel (AWC 1 ) is a $\mathrm{n}_{-} \mathrm{C}_{8} \mathrm{H}_{17}$-decorated foldamer nanotube with an average channel length of $2.8 \mathrm{~nm}$ and a pore diameter of $5.2 \AA$. AWC 1 demonstrates an ultrafast water conduction rate of $1.4 \times 10^{10} \mathrm{H}_{2} \mathrm{O} / \mathrm{s}$ per channel, outperforming the archetypal biological water channel, aquaporin 1, by $27 \%$, while excluding salts (i.e., $\mathrm{NaCl}$ and $\mathrm{KCl}$ ) and protons. Unique to this class of channels, the inwardly facing $\mathrm{C}(\mathrm{sp} 2)-\mathrm{F}$ moieties are proposed as being critical to enabling the ultrafast and superselective water transport properties observed.

\section{INTRODUCTION}

Scarcity of clean water is one of the critical grand challenges facing humanity that currently affects over 4 billion people worldwide $(1,2)$. An important state-of-the-art technology for clean water production and wastewater reuse is reverse-osmosis ( $\mathrm{RO})$ membrane desalination (3). The key to $\mathrm{RO}$ desalination is precise control over transient or fixed sub-nanometer scale passages across the membrane that only allow water molecules to pass through while excluding other solutes like salt ions (4).

In Nature, living organisms regulate transmembrane water flow by membrane-embedded water channels, viz. aquaporins (AQPs). These proteinic channels facilitate superfast water translocation and at the same time completely reject salts and even protons $(5,6)$. For instance, AqpZ, isolated from $E$. coli, features a water transport rate of $\sim 6 \times 10^{9} \mathrm{H}_{2} \mathrm{O} / \mathrm{s}(7,8)$. The other type of AQPs, AQP1 that is present in specific human cells can transport $\sim 1.1 \times 10^{10} \mathrm{H}_{2} \mathrm{O} / \mathrm{s}$ (9), yet with remarkably high water to monovalent ion selectivity over $10^{9}$. Integration of such water-permeating and salt-rejecting AQPS into polymer-based membranes represents an emerging approach for developing the next generation of water desalination and purification technology (10-12). Nevertheless, membrane

\footnotetext{
Department of Chemistry, College of Science, Hainan University, Haikou, Hainan, 570100 China. ${ }^{2}$ Department of Pharmacy, Ludwig Maximilian University Munich Butenandtstraße 5-13, 81377 Munich, Germany. ${ }^{3}$ Department of Physics and Beckman Institute for Advanced Science and Technology, University of Illinois at Urbana-Champaign, Urbana, IL, USA. ${ }^{4}$ McKetta Department of Chemical Engineering, and ${ }^{5}$ Department of Civil, Architectural and Environmental Engineering, The University of Texas at Austin, TX, USA. *Corresponding authors. Emails manish.kumar@utexas.edu (M.K.), aksiment@illinois.edu (A.A.) and 2733910004@qq.com (H.Z.)
}

proteins like AQPs usually suffer from high production costs, challenges with scalability, and questions about structural stability in abiotic environments (13), making them less ideal for large-scale industrial applications.

Motivated by the superior performance of natural AQPs, researchers have expanded extensive effort in developing artificial water channels (AWCs) with simpler structures yet comparable or even exceeding water transport capabilities (14-16). In 2007, Percec and co-workers reported the pioneering work in this field, wherein dendritic dipeptides were employed for the construction of AWCs in lipid membrane (17). Thereafter, various types of unimolecular or self-assembled AWCs have been designed and characterized, including imidazole-quartet (18-23), pillar[n]arenes (24-27), aromatic macrocycles (28), carbon nanotube porins (CNTPs) $(29,30)$, porous organic cages (31), helically folded polymeric nanotubes (32-34), and hydrophilic hydroxyl assemblies (35). The collective conclusion states that water transport efficiency and selectivity highly depend on the geometry and surface chemistry of the channel interior lumen, in which channel-water and water-water interactions occurs, primarily via $\mathrm{H}$-bonds $(9,33,34)$. However, concurrently achieving high single-channel water permeability and high transport selectivity (e.g., rejection of salts and protons) in a single AWC still remains a daunting task to date that has been addressed in only a few studies $(26,34)$.

Here we report on such a high-performance salt- and proton-rejecting AWC system that has a $5.2 \AA$-diameter cavity and transports water at a remarkable rate of $\sim 1.3$ times that of AQP1, outperforming by at least a factor of 4 all other hitherto known salt-rejecting AWCs, except for one very recent example (34). 
A<smiles>[2H]Oc1cc(O)c(C(=O)NN)cc1C(=O)NN</smiles>

$A_{1}, \mathrm{R}=n-\mathrm{C}_{8} \mathrm{H}_{17}$

$A_{2}, \mathrm{R}=n-\left(\mathrm{CH}_{2} \mathrm{CH}_{2} \mathrm{O}\right)_{3} \mathrm{CH}_{3}$

$A_{3}, \mathrm{R}={ }^{i} \mathrm{C}_{4} \mathrm{H}_{9}$

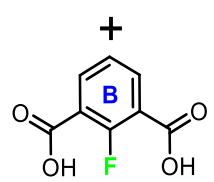

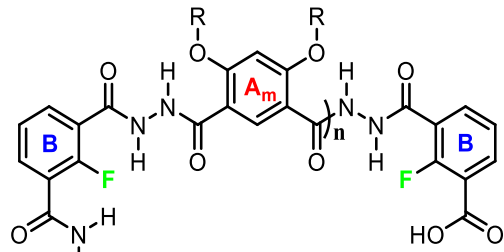

Coupling Reagents

DCM/DMF (5:1) DIEA, 24 h, rt, 50-80\%

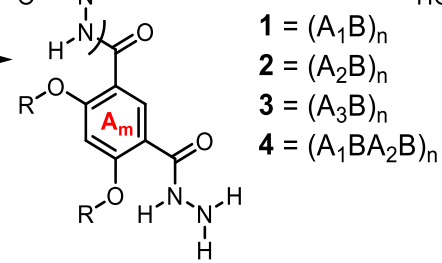

B

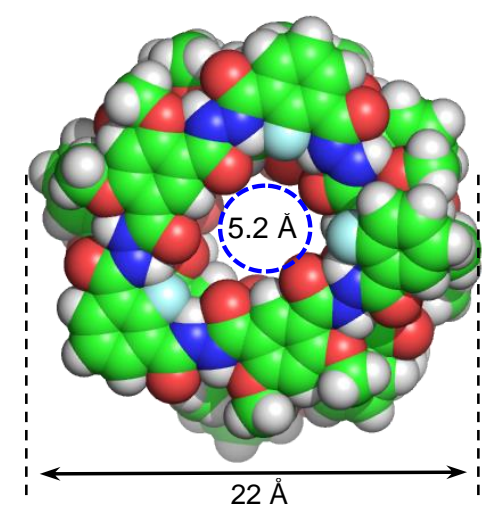

$\mathbf{F}$
C

$$
\mathrm{pH}=7.0
$$

$100 \mathrm{mM} \mathrm{NaCl}$ 200 mM Sucrose

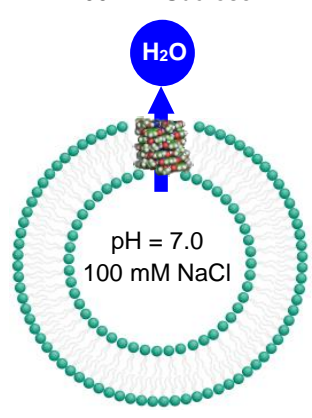

D

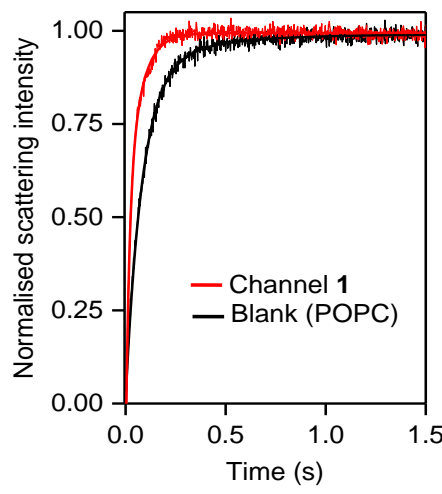

E

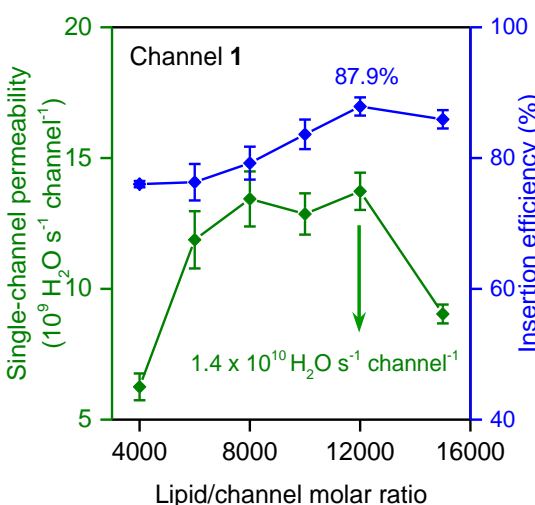

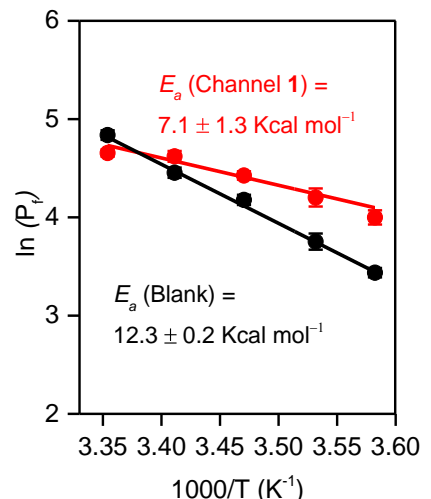

Fig. 1. Molecular structures and water transport properties of fluorofoldamer-based AWCs. A, One-pot copolymerization mediated by amide coupling reagents. B, Quantum mechanics-computed helically folded tubular structures for the AB type copolymers at the HF/6-31G(d) level that gives rise to three $\mathbf{A B}$ units per helical turn; side chains are omitted for clarity. C, Schematic illustration of LUV-based water permeability experiment in presence of $200 \mathrm{mM}$ sucrose as hypertonic solution. D, Representative stopped-flow light scattering traces of blank DOPC LUV of $\sim 120 \mathrm{~nm}$ in diameter and channel 1 reconstituted LUVs under the inwardly directed osmotic gradient. E, Single channel water permeability and insertion efficiency for channel 1 at different lipid/channel molar ratios. F. Arrhenius plots of the water permeability as a function of temperature for DOPC only and channel 1 for determining activation energies $\left(E_{a}\right)$ averaged over three independent runs.

\section{RESULTS AND DISCUSSION Molecular design}

Although the lone pair donation from fluorine, being the most electronegative element in the periodic table, is significantly suppressed, making it a poor $\mathrm{H}$-bond acceptor (36), early studies have established the ability of $\mathrm{C}(s p 2)-\mathrm{F}$ to form weak intramolecular $\mathrm{H}$-bonds in foldamer structures (37-39). Further, fluorine atoms may differ considerably from other $\mathrm{H}$-bond-forming groups in determining the foldamer channel construct and guest binding behaviors (40) by altering the interior pore size and wall smoothness, channel backbone distortion, intermolecular host-guest $\mathrm{H}$-bond interactions, etc. With these concepts in mind, we decided to explore fluorofoldamer-based polymeric hollow channels, having inward-facing fluorine atoms decorating the channel lumen, as possible AWCs.

\section{AWC 1 exhibits the best water transport performance}

Screening a matrix of such AWCs, combinatorically derived from different reaction conditions and monomer structures, culminated in a discovery of the best-performing AWC, water channel 1. AWC 1 is found to conduct water at an ultrafast rate of $1.4 \times 10^{10} \mathrm{H}_{2} \mathrm{O} / \mathrm{s}$ across lipid bilayer membrane, a value that is about 1.3 times higher than that of AQP1 and two times higher than its methoxy-containing analogous channel 1-OMe (see later discussions). Further, the readily synthesized $\mathbf{1}$ also demonstrates near-perfect salt $(\mathrm{NaCl}$ and $\mathrm{KCl})$ and proton rejection, making it an excellent replacement of natural AQPs for possible industrial uses in fabricating next-generation of AWC-based RO membrane for seawater desalination or for use in therapeutics (26).

Synthesis of 1 was carried out by following a previously reported protocol (41). Briefly, a facile one-pot copolymerization reaction between diamine monomer $\mathbf{A}_{1}$ (with $n-\mathrm{C}_{8} \mathrm{H}_{17}$ side chains) and fluoro-containing diacid B using HBTU as the coupling reagent readily produced an off-white powdery product 1 with $\sim 80 \%$ isolated yield (Fig. 1a). Apart from extensive $\pi-\pi$ stacking, intramolecular $\mathrm{H}$-bonds $(\mathrm{R}-\mathrm{O} \cdots \mathrm{H}-\mathrm{N}, \mathrm{C}=\mathrm{O} \cdots \mathrm{H}-\mathrm{N}$ and $\mathrm{C}(s p 2)-\mathrm{F} \cdots \mathrm{H}-\mathrm{N})$ are also expected to stabilize the polymeric product in a helically folded configuration (42). Molecular dynamics (MD) simulations of the pore scaffold shows that the optimized structure exhibits expected helical tubular shape with 

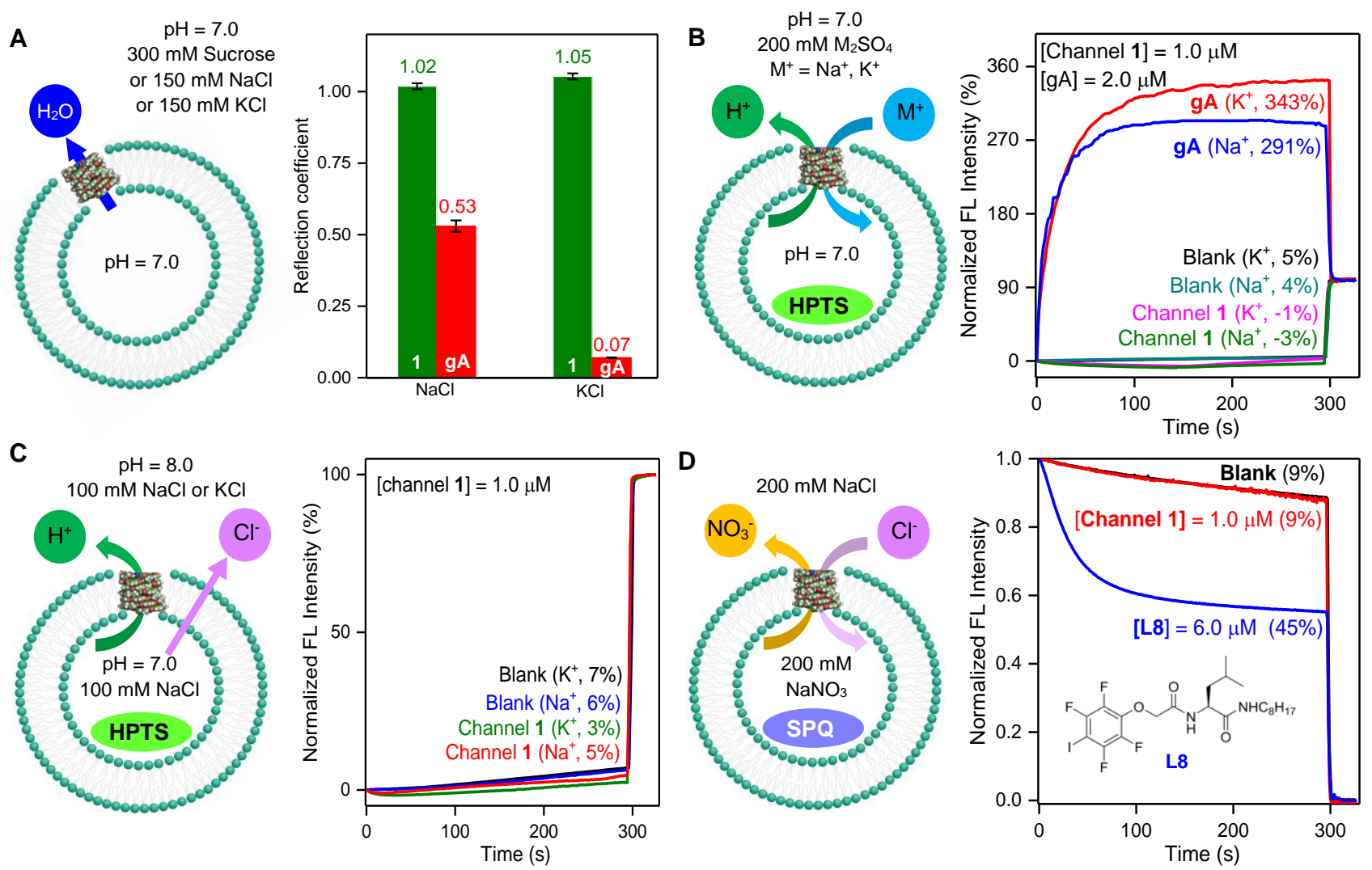

Fig. 2. Ion exclusion properties of channel 1. A, Reflection coefficients calculated from water permeabilities under three types of hypertonic conditions, suggesting high and low salt rejections by $\mathbf{1}$ and gA, respectively. $\mathbf{B}$, pH-sensitive HPTS-based LUV assays under high ionic concentration gradients, confirming that $\mathbf{1}$ does not transport both $\mathrm{Na}^{+}$and $\mathrm{K}^{+}$cations. C, HPTS-based LUV assays under a proton gradient, pointing to an incapability of $\mathbf{1}$ in transporting protons. D, Chloride-sensitive SPQ assay, demonstrating that $\mathbf{1}$ does not transport anions.

inward-facing $\mathrm{C}(s p 2)-\mathrm{F},-\mathrm{N}-\mathrm{H}$ and $-\mathrm{C}=\mathrm{O}$ moieties to the tube lumen. About three $\mathbf{A B}$ repeating units are required to furnish one helical turn, corresponding to a 3.4 A growth in tube length. The inner pore diameter is $\sim 5.2 \AA$ after subtracting the van der Waals radii of the interior atoms. This pore diameter is larger than a water molecule $(2.8 \AA)$, but smaller than first-shell hydrated $\mathrm{Na}^{+}$or $\mathrm{K}^{+}$ions.

The average molecular weight of $\mathbf{1}$ was measured to be $13.9 \mathrm{kDa}$ using gel permeation chromatography (GPC). A NMR-based method was also applied to determine the molecular weight, in which a chiral group was introduced at the amine end of $\mathbf{1}$ as an internal NMR standard (Scheme S3). Based on the area integration ratio of specific ${ }^{1} \mathrm{H}$ signals (Fig. S1), molecular weight of $\mathbf{1}$ was determined as $15.4 \mathrm{kDa}$ (Table S2), agreeing well with the GPC-derived value. Using the simulated pore structure as the guide (three $\mathbf{A B}$ units per helical turn, $\mathrm{MW}$ of $\mathbf{A B}$ unit $=616.7 \mathrm{Da}$; see Fig. $1 \mathrm{~b}$ and Supplementary Table S1), 1 contains 25 AB units in average, measuring at $2.8 \mathrm{~nm}$ in average nanotubular length that is dimensionally comparable to the thickness of typical lipid bilayer membranes (e.g., $2.7 \mathrm{~nm}$ for DOPC) (43). In addition, 1 also displays a characteristic mass pattern with a repeating unit of $617 \mathrm{Da}$ in the MALDI-TOF spectrum (Fig. S2).

The unique structural features of $\mathbf{1}$, including $(I)$ appropriate inner pore diameter intermediate between a water molecule and hydrated ions (e.g., $\mathrm{Na}^{+}$), (ii) membrane-spanning channel length of $2.8 \mathrm{~nm}$, and (iii) special lumen surface chemistry with $\mathrm{H}$-bond donors/acceptors and dipolar C(sp2)-F moieties, lays the structural basis for its water transport property. Stopped flow light-scattering method was employed to quantify the water transport efficiency, using large unilamellar vesicle (LUVs, 120 $\mathrm{nm}$ diameter, Fig. 1c) with channel 1 pre-inserted in the LUV wall $(24,25)$. Under the shrinkage mode, LUVs were exposed to hypertonic buffer solution containing $200 \mathrm{mM}$ sucrose, which induces water efflux and vesicle shrinking. The time-dependent variation of the light-scattering intensity was then captured and analyzed (Fig. 1d), from which water transport rate can be reliably determined. As shown in Fig. 1e, water permeability of $\mathbf{1}$ was largely independent of the lipid to channel molar ratio $(\mathrm{mLCR})$, and the profile peaks at 12000:1, giving water permeability $P_{w}$ of $(41.2 \pm 2.1) \times 10^{-14} \mathrm{~cm}^{3} / \mathrm{s}$. With a channel insertion efficiency of $87.9 \%$ at this $\mathrm{mLCR}$ (Table S3), water permeability translates into a single-channel water transport rate of $(1.4 \pm 0.07) \times 10^{10} \mathrm{H}_{2} \mathrm{O} / \mathrm{s}$, which is $133 \%$ and $27 \%$ faster than the biological AqpZ and AQP1 water channels, respectively (44). The value of $1.4 \times 10^{10} \mathrm{H}_{2} \mathrm{O} / \mathrm{s}$ becomes $0.78 \times$ $10^{10} \mathrm{H}_{2} \mathrm{O} / \mathrm{s}$ using the new equation for $P_{f}$ correction (44).

The water permeability of $\mathbf{1}$-reconstituted LUVs at different temperatures $\left(6-25{ }^{\circ} \mathrm{C}\right)$ were measured, from which its activation energy $E_{a}$ is calculated as $7.1 \pm 1.2 \mathrm{Kcal} \mathrm{mol}^{-1}$ using 
the Arrhenius Equation (Fig. 1f). It is higher than that of the AQPs $\left(\sim 5 \mathrm{Kcal} \mathrm{mol}^{-1}\right)$, but much lower than that from the blank DOPC LUV $\left(12.3 \pm 0.2 \mathrm{Kcal} \mathrm{mol}^{-1}\right)$. In view of the superior water conduction rate of $\mathbf{1}$ compared to AQPs, we assume that low activation energy might not be a necessary feature for highly permeable AWCs, likely because the transport mechanisms differ from that of AQPs in Nature, as proposed before $(33,34)$.

\section{Impact of channel length on water permeability}

Following identical synthetic protocols (41), other amide coupling reagents (HATU, BOP and TBTU) produce the same $\mathbf{A}_{1} \mathbf{B}$ type channels with NMR-derived molecular weights of 20.1, 19.9 and $13.1 \mathrm{kDa}$ (Table S1) that correspond to channel lengths of $4.0,4.0$ and $2.6 \mathrm{~nm}$, respectively. As summarized in Fig. S3, their water transport rates were all found to be lower that of channel 1 (MW = $13.9 \mathrm{kDa}, 2.8 \mathrm{~nm})$. More specifically, at the mLCR of $12000: 1$ and compared to 1 of $2.8 \mathrm{~nm}\left(P_{w}=41.2 \times\right.$ $\left.10^{-14} \mathrm{~cm}^{3} / \mathrm{s}\right), \mathbf{A}_{1} \mathbf{B}$ type channels produced using HATU $(4.0 \mathrm{~nm})$, BOP $(4.0 \mathrm{~nm})$ and TBTU $(2.6 \mathrm{~nm})$ show much lower $P_{w}$ values of $21.9 \times 10^{-14}, 22.0 \times 10^{-14}$ and $32.4 \times 10^{-14} \mathrm{~cm}^{3} / \mathrm{s}$, respectively.

\section{Impact of side chain type on water permeability}

To examine the impact of channel side chains on water transport property, diamine monomers $\mathbf{A}_{2}$ and $\mathbf{A}_{3}$ (carrying $n-\left(\mathrm{CH}_{2} \mathrm{CH}_{2} \mathrm{O}\right)_{3} \mathrm{CH}_{3}$ and ${ }^{i} \mathrm{C}_{4} \mathrm{H}_{9}$ side chains respectively) were also employed in the HBTU-facilitated copolymerization reaction. Their corresponding products $\left(\mathbf{A}_{2} \mathbf{B}\right)_{n}$ and $\left(\mathbf{A}_{3} \mathbf{B}\right)_{n}$ were named channels 2 and $\mathbf{3}$, respectively. Monomers $\mathbf{A}_{\mathbf{1}}$ and $\mathbf{A}_{\mathbf{2}}$ were further pre-mixed in 1:1 ratio, and then stoichiometrically reacted with $\mathbf{B}$ to produce mixed copolymers $\left(\mathbf{A}_{1} \mathbf{B} \mathbf{A}_{2} \mathbf{B}\right)_{\mathrm{n}}$ (e.g., channel 4). From their NMR-derived molecular weights (Supplementary Table 2), the channel tubular lengths can be estimated to be 3.1, 2.9 and $4.1 \mathrm{~nm}$ for $\mathbf{2}, \mathbf{3}$ and $\mathbf{4}$, respectively. At the mLCR of 12000:1, 2 - 4 show much lower $P_{w}$ values of $2.8 \times 10^{-14}, 20.2 \times 10^{-14}$ and $29.4 \times 10^{-14} \mathrm{~cm}^{3} / \mathrm{s}$, respectively. The comparative data among 1-3 indicates the importance of channel side chain lipophilicity on water transport efficiency, and clearly the linear $n-\mathrm{C}_{8} \mathrm{H}_{17}$ represents the best performer for the fluorofoldamer-based AWC scaffold.

\section{High salt and proton rejection capacity of AWC 1}

Besides ultrafast water conduction, the other major challenges for AWCs in mimicking AQP performance are to achieve complete rejection of salts and protons. To this end, we firstly compared the osmotic water permeability $\left(P_{f}\right.$ in $\left.\mathrm{cm} / \mathrm{s}\right)$ values of 1 under three hypertonic conditions (300 mM sucrose, $150 \mathrm{mM}$ $\mathrm{NaCl}$, or $150 \mathrm{mM} \mathrm{KCl}$, Fig. 2a). Since large sucrose molecules are not able to permeate through channel $\mathbf{1}$, the reflection coefficient, defined as $P_{f}(\mathrm{MCl}) / P_{f}$ (sucrose) where $\mathrm{M}^{+}=\mathrm{Na}^{+}$or $\mathrm{K}^{+}$, was used to approximately gauge the transport of salt ions. The well-established dimeric cation-transporting channel gramicidin A ( $\mathrm{gA}$ ) was employed as the positive control, which shows expected reflection coefficients of $0.53 \pm 0.02$ and $0.07 \pm 0.001$ for $\mathrm{NaCl}$ and $\mathrm{KCl}$, arising from its high permeability to both $\mathrm{Na}^{+}$ and $\mathrm{K}^{+}$ions. In contrast, the reflection coefficients of $\mathbf{1}$ were calculated to be $1.02 \pm 0.01$ for $\mathrm{Na}^{+}$and $1.05 \pm 0.01$ for $\mathrm{K}^{+}$at 12000:1 mLCR, confirming the inability of $\mathbf{1}$ to transport either cation across the membrane and its near-perfect salt rejection property (19).

The rejection of $\mathrm{Na}^{+}$and $\mathrm{K}^{+}$cations was further validated by the fluorescence-based HPTS assay, with pH-sensitive HPTS dye molecules entrapped in the LUVs (Fig. 2b). The intravesicular region is set $\mathrm{pH} \mathrm{7,} \mathrm{whereas} \mathrm{the} \mathrm{extravesicular}$ environment is maintained at the same pH but with $200 \mathrm{mM}$ $\mathrm{M}_{2} \mathrm{SO}_{4}(\mathrm{M}=\mathrm{Na}$ or $\mathrm{K})$. Under this high salt gradient, $\mathrm{H}^{+} / \mathrm{M}^{+}$ antiport will increase the $\mathrm{pH}$ of the intravesicular region and hence enhance the HPTS fluorescence intensity. As shown in Fig. 2b, 1 at $1 \mu \mathrm{M}$ was found non-responsive towards $\mathrm{Na}^{+}$or $\mathrm{K}^{+}$ gradient, affirming the impermeability of neither cation through the inner pore of $\mathbf{1}$. On the contrary, gA at the identical channel concentration $(1 \mu \mathrm{M})$ could efficiently transport $\mathrm{Na}^{+}(291 \%)$ and $\mathrm{K}^{+}(343 \%)$ cations. Such observation is in excellent agreement with the reflection coefficient results described earlier, both confirming the inability of $\mathbf{1}$ to transport cations.

The anion transport ability of $\mathbf{1}$ was further examined by using $\mathrm{Cl}^{-}$-sensitive SPQ dye molecules entrapped in LUVs (Fig. 2d). As expected, 1 at $1 \mu \mathrm{M}$ displayed similar SPQ quenching as background $(9 \%)$, whereas the well-established chloride transporter L8 (45) at $6 \mu \mathrm{M}$ (corresponding to $1 \mu \mathrm{M}$ channel concentration) displayed significant decrease $(45 \%)$ in the SPQ fluorescence intensity. In another set of LUV-based experiments where intravesicular region has $100 \mathrm{mM} \mathrm{NaCl}$ at $\mathrm{pH} 7$ and extravesicular region has $67 \mathrm{mM} \mathrm{Na}_{2} \mathrm{SO}_{4}$ at pH 8 (Fig. S5), gA (cation channel, $1 \mu \mathrm{M}$ ), FCCP (proton carrier, $1 \mu \mathrm{M}$ ) and L8 (anion channel, $1.3 \mu \mathrm{M}$ ) induce HPTS fluorescence increase of $56 \%, 22 \%$ and $138 \%$ respectively. In sharp contrast, 1 at $1 \mu \mathrm{M}$ does not cause any fluorescence change.

Proton translocation was probed using the $\mathrm{pH}$ gradient set across the membrane (Fig. 2c). Serving such a purpose, the intra-LUV region contains $100 \mathrm{mM} \mathrm{NaCl}$ at $\mathrm{pH} 7$, whereas the extravesicular region was set $\mathrm{pH} 8$ with $100 \mathrm{mM} \mathrm{NaCl}$ or $\mathrm{KCl}$. If 1 is able to transport protons, the proton efflux (coupled with passive diffusion of cations or anions for charge neutralization) will induce significant $\mathrm{pH}$ increase in the intravesicular region, and dramatic change in HPTS fluorescence intensity will emerge. Experimentally, no fluorescence change was observed at all after addition of $1(1 \mu \mathrm{M})$, suggesting negligible transport of protons. Using a conservative approach (for details, see the Supplementary Section of "Estimation of Proton Transport Rate"), the proton transport rate of $\mathbf{1}$ is estimated to be less than 0.25 proton/s.

Stopped-flow fluorescence analysis was further applied to quantitatively measure chloride permeability through DOPC membrane in the absence and presence of 1 (Fig. S6) $(34,46)$. Based on the determined single-channel $\mathrm{Cl}^{-}$permeability $\mathrm{P}_{\mathrm{Cl}}$ of $(1.7 \pm 07) \times 10^{-20} \mathrm{~cm}^{3} / \mathrm{s}$, the water-to-Cl permselectivity (e.g., $\left.P_{w} / P_{C l}\right)$ for 1 was calculated to be $(2.5 \pm 1.2) \times 10^{7}$. Since $\mathrm{NaCl}$ permeability is limited by the $\mathrm{Na}^{+}$ions in actual desalination processes (46), $2.5 \times 10^{7}$ represents a conservative estimate of the water-to-NaCl permselectivity for $\mathbf{1}$. As compiled in Fig. 3a, this value exceeds the permeability-selective trade-off trendline of current desalination membranes $(11,47)$ by a factor of $\sim 10^{2}$, signifying good potential for developing novel AWC-based desalination membrane that incorporates or is made of $\mathbf{1}$. 
A

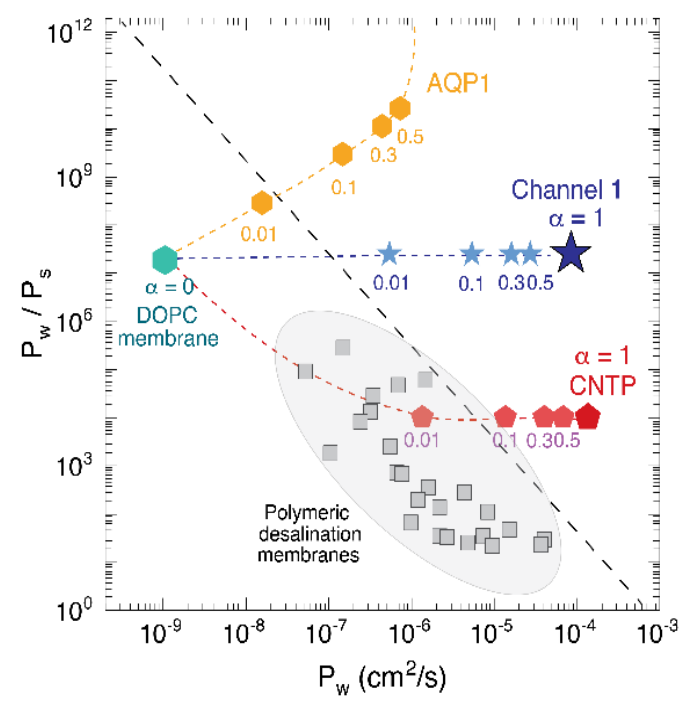

B

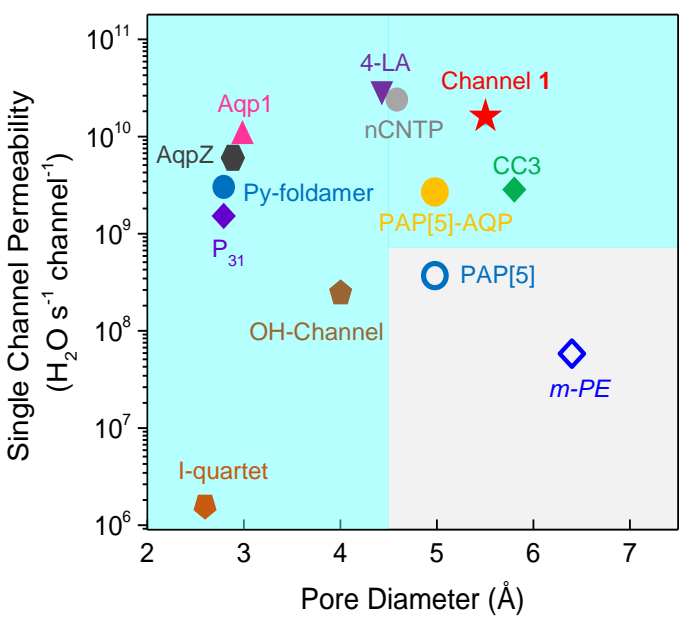

C

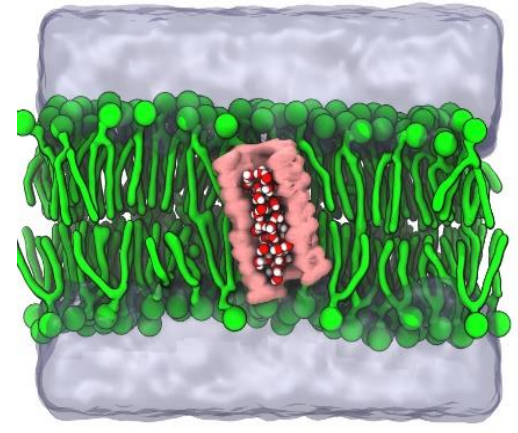

E
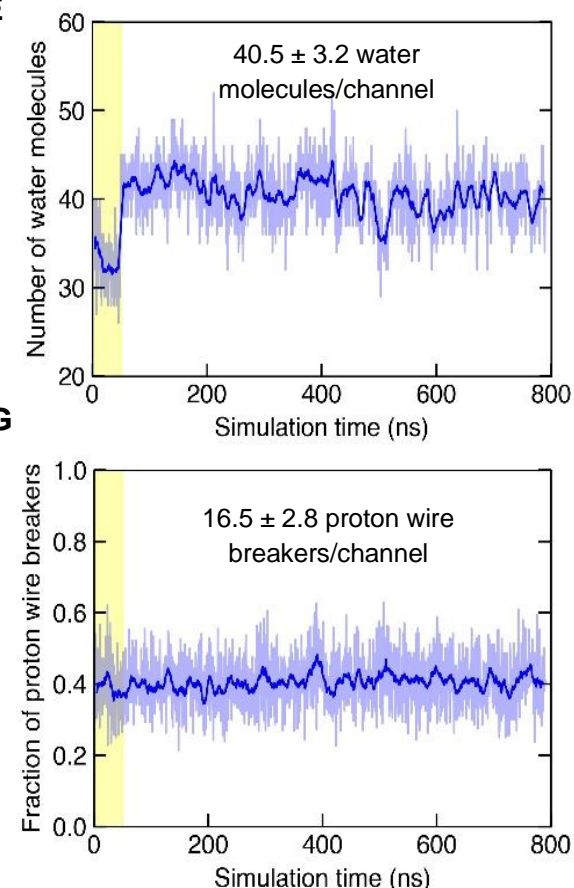

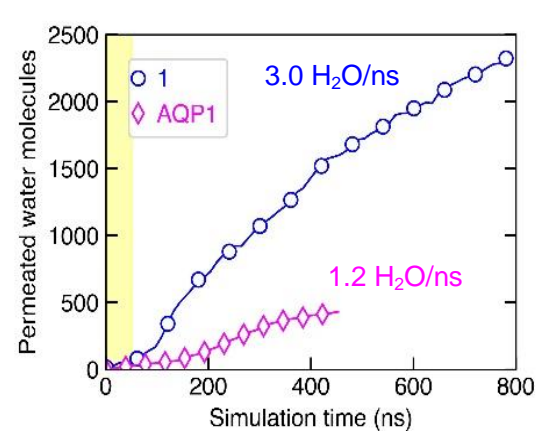

$\mathbf{F}$

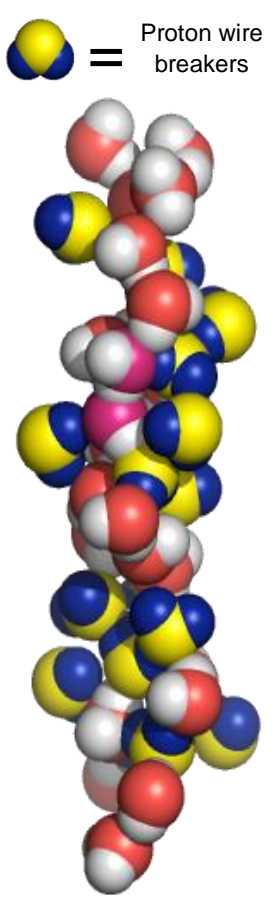

Fig. 3. Ion exclusion and water transport properties of 1, validated using MD simulations, point to its promise as a component of AWC-based desalination membranes. A, Water-to-salt permselectivity values of $\mathbf{1}$, some representative water channels and current polymeric desalination membranes. CNTPs have pore diameter of $\sim 0.47 \mathrm{~nm}$ (52). The $\alpha$ values refer to the fractional membrane volume occupied by water channels (e.g., $\alpha=0.5$ represents the half membrane volume comprises 1 . Dashed line corresponds to upper bound limit in permeability-selectivity value for the current polymer desalination membranes $(11,47)$. B. Single-channel water conduction rate and channel pore diameter of natural AQPs (AqpZ $(7,8)$ and AQP1 $(9,53)$ ) and AWC systems including nCNTP (30), CC3 (31), PAP[5]-AQP (26), 4-LA (34,41), Py-foldamer (33), P31 (32), $\mathrm{OH}$-Channel (35), I-quartet (19), PAP[5] (25) and $m$-PE (28)). Except for non-selective nCNTP, those in the light blue shaded region demonstrate salt-rejecting ability, whereas those in the grey region are ion-conductive. C, A representative snapshot of water-containing channel 1, having 25 AB units and height of $2.8 \mathrm{~nm}$, embedded in POPC membrane and solvated in $1 \mathrm{M}$ aqueous solution of $\mathrm{NaCl}$. $\mathbf{D}$, MD-derived water transport rates for $\mathbf{1}$ and AQP1. The channels were positionally restrained for the first $48 \mathrm{~ns}$ (highlighted region) of each MD trajectory. E, Number of water molecules inside the channel, with a mean of 40.5 water molecules. F, Fraction of proton wire breakers inside the channel, with a mean of $40.7 \%$ ( 16.5 water molecules per channel).G, A water cluster consisting of 40 water molecules out of which 17 serve as proton wire breakers, preventing proton transport via the Grotthuss mechanism.

\section{Comparison with two high-performance AWCs}

As summarized in Fig. 3b, currently there are only two water-transporting systems having higher water conduction rates than both AQP1 $\left(1.1 \times 10^{10} \mathrm{H}_{2} \mathrm{O} / \mathrm{s}\right)(9)$ and AWC $1(1.4 \times$ $\left.10^{10} \mathrm{H}_{2} \mathrm{O} / \mathrm{s}\right)$, i.e., the relatively low selectivity CNT porin $(2.3 \times$ $\left.10^{10} \mathrm{H}_{2} \mathrm{O} / \mathrm{s}\right)(30)$ and the highly selective AWC 4-LA $\left(2.7 \times 10^{10}\right.$ $\mathrm{H}_{2} \mathrm{O} / \mathrm{s}$ ) (34). But it is worth emphasizing that while the water-transporting CNT porin also conducts ions and protons
(30), channel 4-LA requires additional lipid anchors (LA) installed at the helical ends to orient the channel's alignment to achieve the ultrafast water conduction (34). Without such LA modifications, it's water transport rate drastically drops by $75 \%$ to $\sim 0.6 \times 10^{10} \mathrm{H}_{2} \mathrm{O} / \mathrm{s}(34)$, a value that is $\sim 43 \%$ capacity of 1 . Further, it is possible that the LA-enhanced water transport property might deteriorate over time or be altered by the complex environment of a water purification membrane. All 
these make both CNTP and 4-LA potentially less competitive for fabricating practical AWC-based biomimetic water purification membranes than 1 developed in this work $(12,48)$.

\section{Critical roles played by fluorine atoms}

To demonstrate the crucial role of $\mathrm{C}(s p 2)-\mathrm{F}$ moieties in determining the water transport property of channel $\mathbf{1}$, we compared it with the recently reported analogous channel denoted as 1-OMe of $3.0 \mathrm{~nm}$ in height, which differs from 1 in that 1-OMe contains methoxy groups in the positions of F-atoms of 1 (34). As a result of bulky hydrophobic methyl groups helically arranged around the pore interior of $1-\mathrm{OMe}$, its helical backbone is slightly less curved than that of $\mathbf{1}$ having its pore surface decorated by F-atoms. Consequently, the pore diameter of 1-OMe is enlarged to $6.5 \AA$ across, which is larger than 1 (5.2 $\AA$ across). Under the identical conditions, the water transport rate of 1-OMe was determined at $\sim 5 \times 10^{9} \mathrm{H}_{2} \mathrm{O} / \mathrm{s}$ (34), which is $36 \%$ that of 1 . Furthermore, unlike 1 with excellent ion-rejection capability, 1-OMe was permeable to anions (41). Therefore, we speculate that the superior water transport properties of $\mathbf{1}$ should arise from a collection of influencing factors induced by the inward-facing $\mathrm{C}(s p 2)-\mathrm{F}$ moieties, including the smaller atomic size, weak $\mathrm{H}$-bond acceptor ability, dipolar bond characteristics and good hydrophobicity. Further investigative efforts to decipher these factors are currently underway in our laboratory.

\section{Molecular dynamics simulation}

To provide a molecular level explanation of transmembrane water transport through the pore of $\mathbf{1}$ embedded in POPC lipid bilayer membrane, we performed 800 ns long all-atom molecular dynamics (MD) simulations, (Fig. 3c and Supplementary Video 1). To maintain QM-derived diameter of 1 at the MD level, we used the RMSD colvar module of NAMD during the course of MD simulation. Fig. S8 shows the RMSD of 1 as a function simulation time. As the simulation begins, water molecules rapidly start permeating across the lipid bilayer through 1 (Fig. 3d). A linear fit to the water permeation vs simulation time (excluding first $200 \mathrm{~ns}$ ) yields a permeation rate of $\sim 3$ water molecules $/ \mathrm{ns}$, which is higher than 1.2 water molecules/ns for AQP1. At any given instant of time, a water cluster typically having 30-50 water molecules resides inside the channel, with a mean of 40.5 water molecules (Fig. 3e). Among them, $40.7 \%$ or 16.5 water molecules are considered as proton wire breakers (Fig. 3f), which were described and defined in our recent study (34). Interacting with the neighboring water molecules via zero or just one $\mathrm{H}$-bond, or two $\mathrm{H}$-bonds solely via only $\mathrm{O}$-atoms or only $\mathrm{H}$-atoms (Fig. $3 \mathrm{~g}$ ), these breakers prevent forming a continuously $\mathrm{H}$-bonded channelspanning water chain through which protons hop via the Grotthuss mechanism. Interestingly, the breaker type involving the formation of two $\mathrm{H}$-bonds with the adjacent water molecules using only $\mathrm{H}$-atoms is also observed in the NPA motif of AQPs (49). The existence of these proton wire breakers accounts for low proton permeability of $\mathbf{1}$.

Due to the narrow pore, each water molecule forms 1.94
$\mathrm{H}$-bonds with other water molecules inside the channel (Fig. S9a,b) and $0.79 \mathrm{H}$-bonds with the channel wall (Fig. S9c), leading to a total of $2.73 \mathrm{H}$-bonds per water molecule. Taking 4 $\mathrm{H}$-bonds per water molecule $\left(\mathrm{E}_{\mathrm{H} \text {-bond }}=5.1 \mathrm{Kcal} / \mathrm{mol}\right.$ ) in bulk water $(50,51)$, the activation energy for water entry into 1 can be estimated to be $6.5 \mathrm{Kcal} / \mathrm{mol}$, which is consistent with the experimentally determined value of $7.1 \mathrm{Kcal} / \mathrm{mol}$ (Fig. 1f). The fact that $\mathbf{1}$ has a higher activation energy but transports water faster than AQP1 can be largely attributed to its larger pore diameter of $5.2 \AA$ vs $\sim 2.8 \AA$ opening in the central channel of $A Q P 1$ as well as the more than one water wire molecule occupying the pore lumen that differs from the single file transport seen in AQP1 (34).

\section{CONCLUSIONS}

In summary, we have demonstrated ultrahigh water transport efficiency and excellent selectivity of a novel class of fluorofoldamer-based artificial water channels. Produced by facile one-pot copolymerization reaction with good yields, the best-performing water channel 1 of $2.8 \mathrm{~nm}$ in average channel length shows a remarkable water conduction rate of $1.4 \times 10^{10}$ $\mathrm{H}_{2} \mathrm{O} / \mathrm{s}$ and near-perfect rejection of salt ions $\left(\mathrm{Na}^{+}, \mathrm{K}^{+}, \mathrm{Cl}^{-}\right)$and protons. This work uncovers the positive effects of introducing $\mathrm{C}(s p 2)-\mathrm{F}$ moieties on the inner rim of foldamer-based water channel pores, providing a new dimension of channel design principles. This, we believe, will stimulate further development towards the next-generation of membrane technologies for water desalination, nano-filtration and medical dialysis applications.

\section{MATERIALS AND METHODS}

\section{Materials}

All reagents were obtained from commercial suppliers and used as received unless otherwise noted. Aqueous solutions were prepared from MilliQ water. Egg yolk L-a-phosphatidylcholine (EYPC) and 1,2-dioleoyl-sn-glycero-3-phosphocholine lipid (DOPC) were obtained from Avanti Polar Lipids. HEPES, HPTS, SPQ and FCCP refer to 4-(2-hydroxyethyl)-1-piperazine-ethane sulfonic acid, 8-hydroxypyrene1,3,6-trisulfonic acid, 6-methoxy- $\mathrm{N}$-(3-sulfopropyl)quinolinium, carbonyl cyanide-p-trifluoromethoxyphenylhydrazone, respectively.

\section{Water transport study}

In a $2 \mathrm{~mL}$ microcentrifuge tube, $6 \mathrm{mg}$ DOPC $(0.24 \mathrm{~mL}, 25 \mathrm{mg} / \mathrm{mL}$ in $\mathrm{CHCl}_{3}$, Avanti Polar Lipids, USA) and water channel compound (dissolved in $\mathrm{CHCl}_{3}$ ) were mixed at different molar ratios (4000:1 to $15000: 1)$. The solvent was slowly removed by $N_{2}$ flow and the resulting thin film was dried under high vacuum overnight. $1 \mathrm{~mL}$ HEPES buffer (10 mM HEPES, $100 \mathrm{mM} \mathrm{NaCl}, \mathrm{pH}=7.0$ ) was then added into each tube for lipid hydration. In order to maximize incorporation of channel molecules into the lipid bilayer, each microtube was vortexed for $30 \mathrm{~s}$ and sonicated for $150 \mathrm{~s}\left(37 \mathrm{kHz}\right.$, power $\left.100,70^{\circ} \mathrm{C}\right)$ for 10 cycles. A glass spatula was used to scratch down all the lipid residues from the microtube wall to minimize lipid loss and maximize channel incorporation whenever necessary. The lipid/channel mixture was then subjected to 10 freeze-thaw cycles (freezing in liquid $\mathrm{N}_{2}$ for $1 \mathrm{~min}$ and heating at $55{ }^{\circ} \mathrm{C}$ in water bath for $\left.2.5 \mathrm{~min}\right)$. The mixture was then extruded through polycarbonate membrane $(0.1 \mu \mathrm{m})$ at $80{ }^{\circ} \mathrm{C}$ for 15 
times to give LUVs at $6 \mathrm{mg} \mathrm{mL}^{-1}$ lipid concentration. For stopped-flow experiments, this LUV solution was diluted to $1 \mathrm{mg} \mathrm{mL}^{-1}$ with buffer (10 $\mathrm{mM}$ HEPES, $100 \mathrm{mM} \mathrm{NaCl}, \mathrm{pH}=7.0$ ). The LUVs were then exposed to a hypertonic solution (200 mM sucrose, $10 \mathrm{mM}$ HEPES, $100 \mathrm{mM} \mathrm{NaCl}$, $\mathrm{pH}=7.0$ ). During stopped-flow experiment, the abrupt decrease in vesicle size was expected due to transport of water to the extravesicular pool and this event leads to increase in the light scattering intensity of $90^{\circ}$ angle according to the Rayleigh-Gans theory. The changes of light scattering intensity caused by vesicle shrinkage were recorded at a wavelength of $577 \mathrm{~nm}$ and all these plots were fitted in the following form of single exponential function to give rate constant $(k)$ value using the equation shown below:

$$
y=A \exp (-k t)+y 0
$$

where $y$ is change in the light scattering, $k$ is the exponential coefficient of the change in the light scattering and $t$ is time.

With the assumption that change in the light scattering intensity is proportional to the change in the vesicle volume $\left(\Delta \mathrm{V} / \mathrm{V}_{0}\right)$ based on the Boyle-van't Hoff law, the osmotic permeability $\left(P_{f}\right)$ in the unit of $\mathrm{cm} / \mathrm{s}$ was commonly calculated as follow:

$$
\left.P_{f}=k /\left(\left(S / V_{0}\right) \times V_{w} \times \Delta_{o s m}\right)\right)
$$

where $k$ is the exponential coefficient of the change in the light scattering ; $S$ and $V_{0}$ are the initial surface area and volume of the vesicles, respectively; $V_{w}$ is the molar volume of water, and $\Delta_{o s m}$ is the osmolarity difference. The size of LUVs was determined by dynamic light scattering after 10 times dilution of the aforementioned LUVs solution (i.e., $1 \mathrm{mg} \mathrm{mL}^{-1}$ ) with buffer (10 mM HEPES, $100 \mathrm{mM} \mathrm{NaCl}, \mathrm{pH}$ $=7.0$ ).

Following the new approach proposed by Horner and co-workers (44), the water permeability can be alternatively calculated using the new equation shown below:

$$
P_{f}=k \times\left(\mathrm{C}_{\text {in,o }}+\mathrm{C}_{\text {out }}\right) /\left(\left(2 \mathrm{C}_{\text {out }}{ }^{2}\right) \times\left(\left(S / V_{0}\right) \times V_{W}\right)\right)
$$

Where $C_{\text {in, } o}$ and $C_{\text {out }}$ are the osmolytes concentration inside at $\mathrm{t}=0 \mathrm{~s}$ and outside of vesicles, respectively.

To calculate the true water permeability $\left(P_{W}\right.$ in the unit of $\left.\mathrm{cm}^{3} / \mathrm{s}\right)$ of water channels, the $P_{f(b l a n k)}$ value of the blank vesicle without water channels needs to be deducted from $P_{f(\text { channel), which was multiplied by }}$ the vesicle surface area $(S)$ and divided by the number of water channels $(N)$ incorporated in the liposome as shown below. Further taking into consideration of channel incorporation efficiency (CIE, Supplementary Table 3 ), the $P_{w}$ values can be calculated by the following equation:

$$
P_{W}=\left(P_{f(\text { blank })}-P_{f(\text { channel })}\right) \times(S /(\mathrm{N} \times \mathrm{CIE}))
$$

\section{The HPTS assay for cation transport}

EYPC ( $1 \mathrm{~mL}, 25 \mathrm{mg} / \mathrm{mL}$ in $\mathrm{CHCl}_{3}$, Avanti Polar Lipids, USA) was placed in a $10 \mathrm{~mL}$ round bottomed flask and solvent was evaporated by slowly purging $\mathrm{N}_{2}$. After drying the resulting thin film under high vacuum overnight at room temperature, the film was hydrated with a HEPES buffer solution ( $1 \mathrm{~mL}, 10 \mathrm{mM}$ HEPES, $\mathrm{pH}=7.0$ ) containing $\mathrm{pH}$-sensitive HPTS dye molecules $(0.5 \mathrm{mM})$ at room temperature for 1 hour (with occasional vortexing after every 15 minutes) to give a milky suspension. The mixture was then subjected to 10 freeze-thaw cycles (freezing in liquid $\mathrm{N}_{2}$ for 1 minute and heating at $55^{\circ} \mathrm{C}$ in water bath for 2 minutes). The vesicle suspension was extruded through polycarbonate membrane $(0.1 \mu \mathrm{m})$ to produce mostly monodispersed LUVs of about $120 \mathrm{~nm}$ in diameter with HPTS dyes encapsulated inside. The extravesicular HPTS dye was removed by using size exclusion chromatography (stationary phase: Sephadex G-50, GE Healthcare, USA; mobile phase: $10 \mathrm{mM}$ HEPES buffer, $\mathrm{pH}=7.0$ ) and diluted with the mobile phase to yield $3.2 \mathrm{~mL}$ of $10 \mathrm{mM}$ lipid stock solution.

$30 \mu \mathrm{L}$ of HPTS-containing LUVs was added to $1970 \mu \mathrm{L}$ of HEPES buffer (10 mM HEPES, $200 \mathrm{mM} \mathrm{M}_{2} \mathrm{SO}_{4}, \mathrm{pH}=8.0, \mathrm{M}^{+}=\mathrm{Na}^{+}$or $\mathrm{K}^{+}$) in a clean fluorescence cuvette to generate a salt gradient across the lipid bilayer membrane. Fluorescence emission intensity of the HPTS dye, $F_{\mathrm{t}}$, was monitored at $\lambda_{\mathrm{em}}=510 \mathrm{~nm}\left(\lambda_{\mathrm{ex}}=450 \mathrm{~nm}\right)$ with gentle magnetic stirring. Channel 1 dissolved in DMF was added at $t=70 \mathrm{~s}$ and the sample fluorescence was recorded for 300 seconds using fluorescence spectrophotometer (Hitachi, Model F-7100, Japan). At $t=370 \mathrm{~s}, 20 \mu \mathrm{L}$ of $20 \%$ Triton X-100 was added to destroy all vesicles, resulting in destruction of $\mathrm{pH}$ gradient to achieve the maximum change in the HPTS fluorescence intensity. The fluorescence data were also recorded for the dimeric gramicidin A channel under identical experimental condition.

The time axis was normalized according to the following equation: $t$ $=t-70$. The fluorescence intensities $\left(F_{\mathrm{t}}\right)$ were normalized to fractional emission intensity $I_{F}$ using the equation shown below.

$$
I_{\mathrm{F}}=\left[\left(F_{\mathrm{t}}-F_{0}\right) /\left(F_{1}-F_{0}\right)\right] \times 100
$$

whereas $F_{0}=$ fluorescence intensity at $t=0 \mathrm{~s}, F_{1}=$ fluorescence intensity after addition of Triton-X100, and $F_{\mathrm{t}}=$ fluorescence intensity at time $t$.

\section{The HPTS assay for anion transport}

The SPQ-containing LUV suspension $(30 \mu \mathrm{L}, 10 \mathrm{mM}$ lipid, $200 \mathrm{mM}$ $\mathrm{NaNO}_{3}$ ) was added to a $\mathrm{NaCl}$ solution $(1.97 \mathrm{~mL}, 200 \mathrm{mM} \mathrm{NaCl})$ to create a chloride concentration gradient for ion transport observation. A solution of 1 at $120 \mu \mathrm{M}$ in DMSO was then injected into the suspension under gentle stirring. Upon channel addition, the SPQ dye emission was immediately monitored at $430 \mathrm{~nm}$ with excitation at $360 \mathrm{~nm}$ for 300 seconds using fluorescence spectrophotometer (Hitachi, Model F-7100, Japan), after which time an aqueous solution of Triton X-100 $(20 \mu \mathrm{L}$, $20 \% \mathrm{v} / \mathrm{v}$ ) was added to completely eliminate the chloride gradient. The final transport trace was obtained by normalizing the fluorescence intensity using equation shown below.

$$
I_{F}=\left[\left(F_{\mathrm{t}}-F_{0}\right) /\left(F_{1}-F_{0}\right)\right] \times 100
$$

Where $F_{0}=$ fluorescence intensity just before the channel addition (at $t=$ $0 \mathrm{~s}), F_{\mathrm{t}}=$ Fluorescence intensity at time $t$, and $F_{1}=$ fluorescence intensity after addition of Triton-X100.

\section{Activation energy measurements}

To determine activation energies for water transport, we conducted water permeability measurements at different temperatures at intervals of $5{ }^{\circ} \mathrm{C}$ between 20 and $40{ }^{\circ} \mathrm{C}$. For these experiments, the solution reservoir and the measurement cell of the stopped-flow instrument were maintained at a set temperature by a recirculating heater/chiller (Polystat, Cole Parmer). Permeability rates through channels at varying temperatures were used to construct an Arrhenius plot.

$$
\operatorname{Ln}(k)=\operatorname{Ln}(A)-E a /(R T)
$$

where $k$ is the exponential coefficient of the change in the light scattering; $A$ is pre-exponential factor; Ea is activation energy; $R$ is gas constant; $T$ is absolute temperature in Kelvin.

\section{Molecular dynamics simulations}

All MD simulations were performed using the MD program NAMD2 with periodic boundary conditions and using the particle mesh Ewald (PME) method to calculate the long-range electrostatics. The Nose-Hoover Langevin piston and Langevin thermostat were used to maintain the constant pressure and temperature in the system. CHARMM36 force field parameters describe the bonded and non-bonded interactions of among, lipid bilayer membranes, water and ions. An 8-10-12 A cutoff scheme was used to calculate van der Waals and short range electrostatics forces. All simulations were performed using a 2 fs time step to integrate the equation of motion. SETTLE algorithm was applied to keep water molecules rigid whereas RATTLE algorithm constrained 
all other covalent bonds involving hydrogen atoms. The coordinates of the system were saved at an interval of $19.2 \mathrm{ps}$. The analysis and post processing the simulation trajectories were performed using VMD and CPPTRAJ.

The initial structure of channel 1 having 25 AB repeating units was built using a fragment-assembly strategy. Specifically, a helical fragment containing $8 \mathbf{A B}$ repeating units was built using Gaussview and optimized at the $\mathrm{HF} / 6-31 \mathrm{G}(\mathrm{d})$ level. Based on the optimized structural parameters (bond angle/length, dihedral angle, etc) of this helical fragment, we then built longer channel 1. The topology and force field parameters for the monomeric unit of 1 were created using the CHARMM general force fields (CGenFF) webserver. Subsequently, the channel was embedded into a $10.5 \times 10.5 \mathrm{~nm}^{2}$ patch of pre-equilibrated POPC lipid bilayer membrane. The lipid patch was generated using the CHARMM-GUI membrane builder and pre-equilibrated for approximately $400 \mathrm{~ns}$. Lipid molecules that overlapped with the channel were removed. The system was then solvated with water using the Solvate plugin of VMD. Sodium and chloride ions were added to $0.6 \mathrm{M}$ concentration using the Autoionize plugin of VMD. The final assembled system measured $10.5 \times 10.5 \times 9.0 \mathrm{~nm}^{3}$ and contained 100,482 atoms.

Following the assembly, the system underwent 1200 steps of energy minimization using the conjugate gradient method to remove steric clashes. After energy minimization, the system was subjected to a $48 \mathrm{~ns}$ equilibration at a constant number of atoms $(N)$, pressure $(P=1$ bar $)$ and temperature $(\mathrm{T}=300 \mathrm{~K})$, the NPT ensemble, with harmonic restraints applied to all non-hydrogen atoms of channels that surrounded the transmembrane pore. The restraints were applied relative to the initial coordinates of the atoms, with spring constants at 1 $\mathrm{kcal} \mathrm{mol}{ }^{-1} \AA^{-2}$. After $48 \mathrm{~ns}$, the harmonic restraints were removed, and the system was equilibrated while restraining the RMSD of the channel with respect to its QM optimized initial conformation using the colvar module of NAMD. For the corresponding references, see the supplementary information.

\section{SUPPLEMENTARY MATERIALS}

Supplementary material for this article is available at http://advances.sciencemag.org/

\section{REFERENCES AND NOTES}

1. M. M. Mekonnen, A. Y. Hoekstra, Four billion people facing severe water scarcity. Sci. Adv. 2, e1500323 (2016).

2. A. Y. Hoekstra, T. O. Wiedmann, Humanity's unsustainable environmental footprint. Science 344, 1114 (2014).

3. J. Imbrogno, G. Belfort, Membrane Desalination: Where Are We, and What Can We Learn from Fundamentals? Annu. Rev. Chem. Biomol. Eng. 7, 29-64 (2016).

4. T. E. Culp et al., Nanoscale control of internal inhomogeneity enhances water transport in desalination membranes. Science $\mathbf{3 7 1}$, 72-75 (2021).

5. K. Murata et al., Structural determinants of water permeation through aquaporin-1. Nature 407, 599-605 (2000).

6. E. Tajkhorshid et al., Control of the Selectivity of the Aquaporin Water Channel Family by Global Orientational Tuning. Science 296, 525 (2002).

7. M. J. Borgnia, D. Kozono, G. Calamita, P. C. Maloney, P. Agre, Functional reconstitution and characterization of AqpZ, the E. coli water channel protein11Edited by W. Baumeister. J. Mol. Biol. 291, 1169-1179 (1999).

8. A. Horner, C. Siligan, A. Cornean, P. Pohl, Positively charged residues at the channel mouth boost single-file water flow. Faraday Discuss. 209, 55-65 (2018).
9. A. Horner et al., The mobility of single-file water molecules is governed by the number of $\mathrm{H}$-bonds they may form with channel-lining residues. Sci. Adv. 1, e1400083 (2015).

10. M. Kumar, M. Grzelakowski, J. Zilles, M. Clark, W. Meier, Highly permeable polymeric membranes based on the incorporation of the functional water channel protein Aquaporin Z. Proc. Natl. Acad. Sci. USA 104, 20719-20724 (2007).

11. H. B. Park, J. Kamcev, L. M. Robeson, M. Elimelech, B. D. Freeman, Maximizing the right stuff: The trade-off between membrane permeability and selectivity. Science 356, eaab0530 (2017).

12. E. Abaie, L. Xu, Y.-X. Shen, Bioinspired and biomimetic membranes for water purification and chemical separation: A review. Front. Environ. Sci. Eng. 15, 124 (2021).

13. J. R. Werber, C. O. Osuji, M. Elimelech, Materials for next-generation desalination and water purification membranes. Nat. Rev. Mater. 1, 16018 (2016).

14. Y. P. Huo, H. Q. Zeng, "Sticky"-Ends-Guided Creation of Functional Hollow Nanopores for Guest Encapsulation and Water Transport. Acc. Chem. Res. 49, 922-930 (2016).

15. B. Gong, Artificial water channels: inspiration, progress, and challenges. Faraday Discuss. 209, 415-427 (2018).

16. L.-B. Huang, M. Di Vincenzo, Y. Li, M. Barboiu, Artificial Water Channels: Towards Biomimetic Membranes for Desalination. Chem. Eur. J. 27, 2224-2239 (2021).

17. M. S. Kaucher et al., Selective Transport of Water Mediated by Porous Dendritic Dipeptides. J. Am. Chem. Soc. 129, 11698-11699 (2007).

18. Y. Le Duc et al., Imidazole-Quartet Water and Proton Dipolar Channels. Angew. Chem. Int. Ed. 50, 11366-11372 (2011).

19. E. Licsandru et al., Salt-Excluding Artificial Water Channels Exhibiting Enhanced Dipolar Water and Proton Translocation. J. Am. Chem. Soc. 138, 5403-5409 (2016).

20. I. Kocsis et al., Oriented chiral water wires in artificial transmembrane channels. Sci. Adv. 4, eaao5603 (2018).

21. M. Di Vincenzo et al., Biomimetic artificial water channel membranes for enhanced desalination. Nature Nanotech., (2020).

22. M. Di Vincenzo et al., Tunable membranes incorporating artificial water channels for high-performance brackish/low-salinity water reverse osmosis desalination. Proc. Natl. Acad. Sci. U. S. A. 118 e2022200118 (2021).

23. L.-B. Huang et al., Bilayer versus Polymeric Artificial Water Channel Membranes: Structural Determinants for Enhanced Filtration Performances. J. Am. Chem. Soc. 143, 14386-14393 (2021).

24. X.-B. Hu, Z. Chen, G. Tang, J.-L. Hou, Z.-T. Li, Single-Molecular Artificial Transmembrane Water Channels. J. Am. Chem. Soc. 134, 8384-8387 (2012)

25. Y.-x. Shen et al., Highly permeable artificial water channels that can self-assemble into two-dimensional arrays. Proc. Natl. Acad. Sci. U.S.A. 112, 9810 (2015).

26. Z.-J. Yan et al., Artificial Aquaporin That Restores Wound Healing of Impaired Cells. J. Am. Chem. Soc. 142, 15638-15643 (2020).

27. D. Strilets et al., Biomimetic Approach for Highly Selective Artificial Water Channels Based on Tubular Pillar[5]arene Dimers. Angew. Chem. Int. Ed. 59, 23213-23219 (2020).

28. X. Zhou et al., Self-assembling subnanometer pores with unusual mass-transport properties. Nat. Commun. 3, 949 (2012).

29. R. H. Tunuguntla, F. I. Allen, K. Kim, A. Belliveau, A. Noy, Ultrafast proton transport in sub-1-nm diameter carbon nanotube porins. Nat. Nanotechnol. 11, 639-644 (2016). 
30. R. H. Tunuguntla et al., Enhanced water permeability and tunable ion selectivity in subnanometer carbon nanotube porins. Science 357, 792-796 (2017).

31. Y. D. Yuan et al., Porous organic cages as synthetic water channels. Nat. Commun. 11, 4927 (2020).

32. J. Shen et al., Polypyridine-Based Helical Amide Foldamer Channels: Rapid Transport of Water and Protons with High Ion Rejection. Angew. Chem. Int. Ed. 59, 13328-13334 (2020).

33. J. Shen et al., Aquafoldmer-Based Aquaporin-like Synthetic Water Channel. J. Am. Chem. Soc. 142, 10050-10058 (2020).

34. A. Roy et al., Foldamer-based ultrapermeable and highly selective artificial water channels that exclude protons. Nat. Nanotechnol. 16, 911-917 (2021).

35. L.-B. Huang et al., Hydroxy Channels-Adaptive Pathways for Selective Water Cluster Permeation. J. Am. Chem. Soc. 143, 4224-4233 (2021).

36. D. O'Hagan, Understanding organofluorine chemistry. An introduction to the C-F bond. Chem. Soc. Rev. 37, 308-319 (2008).

37. C. Ren et al., Crystallographic Realization of the Mathematically Predicted Densest All-Pentagon Packing Lattice by C5-Symmetric "Sticky" Fluoropentamers. Angew. Chem. Int. Ed. 50, 10612-10615 (2011).

38. Q. Gan et al., Quadruple and Double Helices of 8-Fluoroquinoline Oligoamides. Angew. Chem. Int. Ed. 47, 1715-1718 (2008).

39. C. L. Ren et al., Crystallographic realization of the mathematically predicted densest "All Pentamer" packing lattice by C5-symmetric "sticky" fluoropentamers. Angew. Chem., Int. Ed. 50, 10612-10615 (2011).

40. C. Li et al., F $\cdots \mathrm{H} \quad \mathrm{N}$ Hydrogen Bonding Driven Foldamers: Efficient Receptors for Dialkylammonium lons. Angew. Chem. Int. Ed. 44, 5725-5729 (2005).

41. A. Roy et al., Polyhydrazide-Based Organic Nanotubes as Efficient and Selective Artificial lodide Channels. Angew. Chem. Int. Ed. 59, 4806-4813 (2020).

42. S. K. Mishra, N. Suryaprakash, Intramolecular hydrogen bonds involving organic fluorine in the derivatives of hydrazides: an NMR investigation substantiated by DFT based theoretical calculations. Phys. Chem. Chem. Phys. 17, 15226-15235 (2015).

43. Y. Guo, S. Pogodin, V. A. Baulin, General model of phospholipid bilayers in fluid phase within the single chain mean field theory. $J$. Chem. Phys 140, 174903 (2014).

44. C. Hannesschläger, T. Barta, C. Siligan, A. Horner, Quantification of Water Flux in Vesicular Systems. Sci. Rep. 8, 8516 (2018).

45. C. Ren et al., A halogen bond-mediated highly active artificial chloride channel with high anticancer activity. Chem. Sci. 9, 4044-4051 (2018).

46. W. Song et al., Artificial water channels enable fast and selective water permeation through water-wire networks. Nature Nanotech. 15, 73-79 (2020).

47. J. R. Werber, M. Elimelech, Permselectivity limits of biomimetic desalination membranes. Sci. Adv. 4, eaar8266 (2018).

48. W. Song, M. Kumar, Artificial water channels: toward and beyond desalination. Curr. Opin. Chem. Eng. 25, 9-17 (2019).

49. U. Kosinska Eriksson et al., Subangstrom Resolution X-Ray Structure Details Aquaporin-Water Interactions. Science $\mathbf{3 4 0}$, 1346-1349 (2013).

50. C. Zhang, J. Wu, G. Galli, F. Gygi, Structural and Vibrational Properties of Liquid Water from van der Waals Density Functionals. J. Chem. Theory Comput. 7, 3054-3061 (2011).

51. Y. Li et al., Water-ion permselectivity of narrow-diameter carbon nanotubes. Sci. Adv. 6, eaba9966 (2020).
52. V. Freger, Selectivity and polarization in water channel membranes: lessons learned from polymeric membranes and CNTs. Faraday Discuss. 209, 371-388 (2018).

53. P. Pohl, S. M. Saparov, M. J. Borgnia, P. Agre, Highly selective water channel activity measured by voltage clamp: Analysis of planar lipid bilayers reconstituted with purified AqpZ. Proc. Natl. Acad. Sci. U.S.A. 98, 9624 (2001).

\section{Acknowledgements}

Funding: This work was supported by Hainan University, China, the National Science Foundation (USA) under grant DMR-1827346, the National Institutes of Health under grant P41-GM104601. The work in MK lab was supported by the US National Science Foundation under grants CBET 1946392 and CBET 1952295. Supercomputer time was provided through the Leadership Resource Allocation MCB20012 on Frontera of the Texas Advanced Computing Center and XSEDE allocation no. MCA05S028. Author Contributions: J. S. performed water and ion transport study. A. R. carried out the synthesis of polymers and some ion transport study. R. $Y$ carried out some water transport study. H. J. and A. A. conducted molecular dynamics analyses of water transport properties. L. S., Y. T., W. S., M. S. and M. K. carried out chloride permeability measurement. $H$. Z. conceived and supervised the project and wrote the manuscript. All authors participated in discussion and editing of the manuscript. Competing interests: The authors declare no competing financial interests. Data and materials availability: All data needed to evaluate the conclusions in the paper are present in the paper and/or the Supplementary Materials. Additional data related to this paper may be requested from the authors. 